\title{
Sonoelastographic Features of the Patellar Ligament in Clinically Normal Dogs
}

\author{
Angela P. Piccionello ${ }^{1}$ Daniele Serrani ${ }^{1}$ Valeria Busoni ${ }^{2}$ Alberto Salvaggio ${ }^{1}$ Mattia Bonazzi ${ }^{3}$ \\ Chiara Bergamino ${ }^{4}$ Antonella Volta ${ }^{5}$
}

${ }^{1}$ School of Bioscience and Veterinary Medicine, University of Camerino, Matelica, Italy

2 Department of Imaging, Faculté de Médicine Vétérinaire, Université de Liège, Liège, Belgium

${ }^{3}$ Casa di Cura Veterinaria San Geminiano, Modena, Italy

${ }^{4}$ Department of Diagnostic Imaging, School of Veterinary Medicine, University College Dublin, College of Agriculture Food Science and Veterinary Medicine, Dublin, Ireland

${ }^{5}$ Department of Veterinary Science, University of Parma, Parma, Italy

Address for correspondence Daniele Serrani, DVM, MSVM, MRCVS School of Bioscience and Veterinary Medicine, University of Camerino, Via Circonvallazione 93, Matelica (MC) 62024, Italy (e-mail: d.serrani89@gmail.com).

Vet Comp Orthop Traumatol 2018;31:279-284.

\begin{abstract}
Keywords

- patellar ligament

- desmopathy

- sonoelastography

- dog

Objective This article describes the sonoelastographic features of the patellar ligament of sound dogs and tests feasibility, reproducibility and repeatability.

Methods Clinically healthy medium-to-large breed dogs were enrolled. Sonoelastographic images of the patellar ligaments were obtained in lateral recumbency with the stifle flexed by an experienced operator and by a senior veterinary student. The elasticity colour map included red (soft), green (intermediate) and blue (hard). Tissue elasticity was measured by calculating the percentage of softness with dedicated software. Categorical, qualitative data analysis was performed using a weighted kappa statistic for repeatability and reproducibility. A categorical qualitative assessment was performed based on a grading scale of 1 to 5 (soft, mostly soft, intermediate, mostly hard and hard).

Results Fourteen clinically normal dogs were considered. A total of 28 patellar ligaments were examined. Overall, 25 of the patellar ligaments were graded as soft or mostly soft and the remaining 3 as intermediate. Repeatability was $86.2 \%$, with a weighted kappa of 0.64 (good), for the well-trained sonographer and $83.3 \%$, with a weighted kappa of 0.53 (moderate), for the senior student. Reproducibility was $86.2 \%$, with a weighed kappa of 0.65 (good).

Clinical Significance Sonoelastography of the canine patellar ligament is a feasible and reproducible technique. Patellar ligaments in clinically normal dogs showed highly elastic biomechanical properties.
\end{abstract}

\section{Introduction}

Desmopathy of the patellar ligament in dogs has often been reported after tibial plateau levelling osteotomy and tibial tuberosity advancement. ${ }^{1-5}$ Patellar ligament desmopathy can result in an asymptomatic thickening on postoperative radiographs or have clinical relevance. It generally appears self-limiting, but several cases can cause marked lameness and prolonged recovery from tibial plateau levelling osteotomy and tibial tuberosity advancement. . $^{2-4}$

The diagnosis of patellar ligament desmopathy is primarily clinical, but an accurate assessment of the injury can be challenging. Even if radiography can detect soft tissue swelling, irregular margins and thickening of the patellar ligament at the level of the tibial tuberosity, ultrasound received

November 6, 2017

accepted after revision

March 26, 2018

published online

June 11, 2018 (c) 2018 Georg Thieme Verlag KG Stuttgart · New York
DOI https://doi.org/ 10.1055/s-0038-1651499. ISSN 0932-0814. 
examination and magnetic resonance imaging are often used to better assess the injury. Ultrasound examination is costeffective and widespread and has the advantage of dynamic capability compared with magnetic resonance imaging. ${ }^{6}$

Patellar ligament and Achilles tendon lesions have previously been investigated with ultrasound examination and include thickening, disruption of fibres orientation, hypoechoic or anechoic core lesions and increased echogenicity of periligamentous tissues. ${ }^{6-12}$ Although ultrasound examination provides qualitative evaluations, it does not enable testing of the mechanical and functional proprieties of the structures examined; therefore, it is not possible to clearly quantify the severity of the lesion. ${ }^{13}$ A non-quantitative method, using conventional magnetic resonance imaging or sonography, has therefore been proposed. ${ }^{13}$

Sonoelastography is a recent non-invasive, ultrasoundbased technique that evaluates the mechanical properties of tissues. ${ }^{14}$ Sonoelastography is based on the principle that tissue displacement, in response to external compression, produces 'strain' within the tissue, and the strain is lower in harder tissue than in softer tissue. ${ }^{14,15}$ The strain is defined as the change in shape of a tissue, while a stress, a force acting on unit area, is applied on it. The elasticity of a material describes the tendency of a tissue to resume its original shape after being deformed by a stress. ${ }^{16}$ Sonoelastography evaluates tissue motion by comparing the echoes before and after tissue compression. The elastic modulus (a physical quantity measuring stiffness) is proportional to stiffness and inversely proportional to tissue strain. ${ }^{16}$ This strain information can be presented either as a visual map (grey scale or colour-coded) or as a semi-quantitative strain ratio (measurements of strain differences between two user-defined areas). ${ }^{16}$

In humans, several clinical studies applying strain ratios in differentiating malignant from benign lesions of the breast, thyroid, prostate, testicles, liver, ovaries, cervix and pancreas have reported promising results. ${ }^{17-28}$ This technique is useful for assessing tendinopathies of the Achilles tendon and the patellar ligament. ${ }^{7,29-33}$ In fact, the different elasticity patterns of normal and diseased tendons correlate well with histologic degeneration. ${ }^{34}$

The relationship between elasticity and tendon strength has already been investigated, and it has been demonstrated that detecting non-invasively the elastic module of a tendon with ultrasounds, it is possible to predict the ultimate mechanical properties of a tendon. ${ }^{35}$

In veterinary medicine, sonoelastography is a feasible, repeatable and reproducible technique for estimating tissue stiffness in canine and feline liver, spleen and kidneys and in canine prostate. ${ }^{36,37}$

Furthermore, sonoelastography is a feasible technique to evaluate normal and diseased digital flexor tendons in horses ${ }^{38,39}$ and is well correlated with magnetic resonance imaging and ultrasonographic investigation. ${ }^{39}$ Moreover, as tendon failure is highly strain dependent, ${ }^{35}$ sonoelastography may also have a prognostic value by assessing the evolution of the disease.

To our knowledge, there are no published reports about the application of sonoelastography in the canine patellar ligament. Based on comparative studies in humans and horses, we hypothesized that sonoelastography could be useful in the evaluation of the canine patellar ligament. In this context, the aims of this study were as follows:

- To describe the sonoelastographic features of the patellar ligament in clinically healthy, medium-to-large breed dogs.

- To evaluate the feasibility, the repeatability and the reproducibility of sonoelastography of the canine patellar ligament.

This would provide a basis for future studies of canine patellar ligament diseases.

\section{Materials and Methods}

Clinically healthy dogs of a variety of ages and breeds were prospectively recruited. In all cases, the owners provided an informed consent for the procedure, and the protocol was reviewed and accepted by the local ethical committee. Before enrolment, each dog underwent physical and complete orthopaedic examinations and haematology and serum biochemical analyses. Only normal dogs were included in the study. Conventional B-mode ultrasonographic examination of both patellar ligaments was performed (-Fig. 1) without sedation, using a My Laboratory Class C ultrasound machine (Esaote; Genova, Italy) equipped with a 12 to $18 \mathrm{MHz}$ linear transducer (LA 435; Esaote, Genova, Italy). The region was shaved and coupling gel was applied. A patellar ligament was considered normal when the fibrillar echotexture was homogeneous and parallel and slightly broadened at origin. Ligaments that exhibited ultrasonographic evidence of pathologies, such as disrupted patterns, increased cross-sectional diameter or internal mineralisation, were excluded. Dogs were also excluded if the surface area of the probe in contact to the skin (footprint) was too long and protruded across the patella, the tibial tuberosity, or both, preventing complete contact

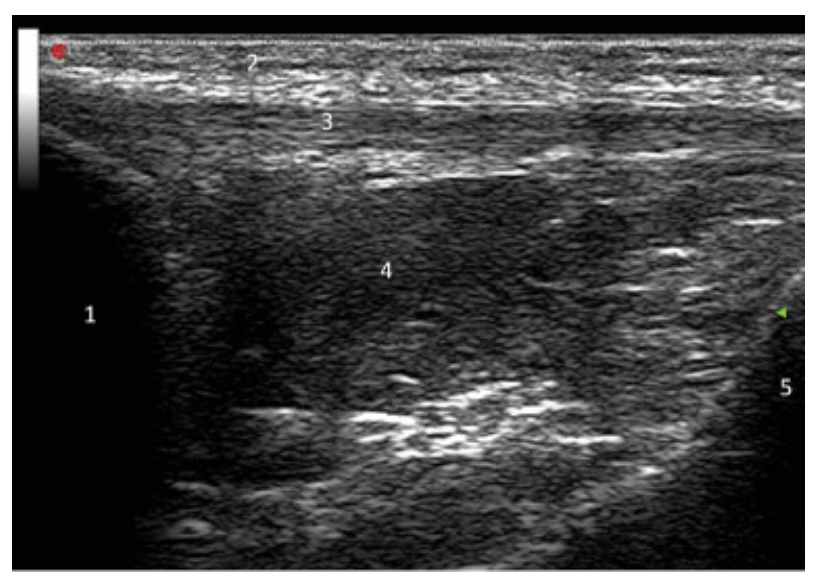

Fig. 1 B-mode longitudinal ultrasonographic image of a normal patellar ligament in a 2-year-old female Vizsla dog. Proximal is to the left, 1: distal extremity of the patella, 2: cutaneous/subcutaneous tissue, 3: patellar ligament, 4: infrapatellar fat pad, and 5: tibial plateau. 
with the patellar ligament. Sonoelastography was then performed with the same machine equipped with a dedicated software (ElaXto, Esaote), without sedation, by two different operators: a well-trained ultrasonographer and a senior veterinary student. For conventional ultrasonographic and sonoelastographic examinations, dogs were positioned in lateral recumbency with the stifle in maximal passive flexion, to avoid the anisotropy of the fibrillar structures of the ligament. ${ }^{40}$ The elastosonographic images were obtained by applying light rhythmic pressure with the probe. Only longitudinal sections were acquired, and the operators evaluated each patellar ligament twice. Investigation was made blindly, and each operator was not aware about the results of the acquisition of the other operator.

The elastosonographic images were characterized by a colour translucent map superimposed on the B-mode images, including the subcutis, the patellar ligament and the infrapatellar fat pad. Each colour indicated the relative elasticity of the different structures compared with the mean elasticity of the entire area: blue (mostly hard), green (intermediate) and red (soft) (-Fig. 2). Only images without artefacts were evaluated. Images were classified as diagnos-

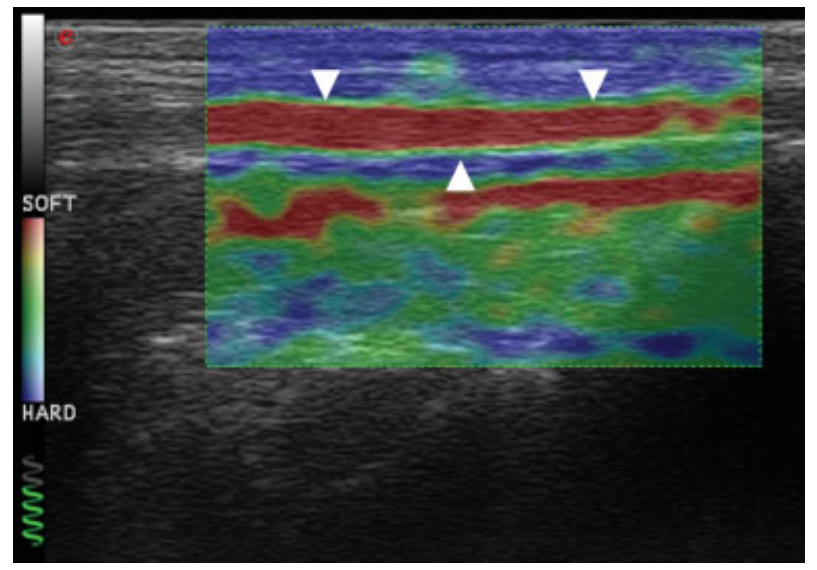

Fig. 2 Elastogram of a normal patellar ligament of a 1-year-old male Labrador retriever dog. The cutis/subcutis is mainly blue (hard), the main body of the ligament (arrowheads) is homogeneously red (soft) with an intermediate (green) peritendinous tissue, and the infrapatellar fat pad is mainly intermediate (green) with red (soft) striations. tic when (1) the real-time visual indicator provided by the software determined an adequate degree of correlation of the relative hardness over time (green coil); (2) the distribution of colours in the elastogram compared to the underlying B-mode image was coherent (overlying dermal layer appeared mostly hard, while the underlying fat pad was mostly intermediate with soft striations). The images were then recorded and stored. Each elastogram was subsequently reviewed, and the images were processed by means of the aforementioned software ElaXto. Using the superimposed Bmode image as a guide, two different types of region of interest (ROI) were drawn: a ROI that covered the entire main body of the ligament (excluding the patellar and tibial origin) and a smaller ROI in the central area of each ligament (-Fig. 3). The softness of the tissue was measured quantitatively using the percentage of softness of the tissue in the ROI (Elx-t\%sft).

Categorical, qualitative data analysis was performed using a weighted kappa statistic for repeated evaluations by the same (repeatability) and by a different observer (reproducibility). For that purpose, the categorical qualitative assessment was performed translating the percentage of softness into a grading scale of 1 to 5 ( $1=$ soft; $2=$ mostly soft; 3 = intermediate; $4=$ mostly hard; $5=$ hard). Values were assigned as reported in - Table 1. Categorical qualitative assessment was performed in the same way for the two different types of ROI measurements (entire main body of the ligament vs. central part).

Statistical significance was set at $p<0.05$. For statistical analyses, we used Excel, (Microsoft; Redmond, Washington, United States) and GraphPad Quick Calcs Software (c2016.

\section{Results}

We examined 30 patellar ligaments from 15 clinically healthy dogs, 8 males and 7 females, belonging to different breeds of medium- and large-sized dogs and aged 1 to 11 years (age average \pm standard deviation $=5.03 \pm 3.71$; - Supplementary Appendix Table 1, availablein online version only). One dog was excluded due to mineralization within both patellarligaments.

The results of the sonoelastography of the remaining 28 normal patellar ligaments were subjectively examined for
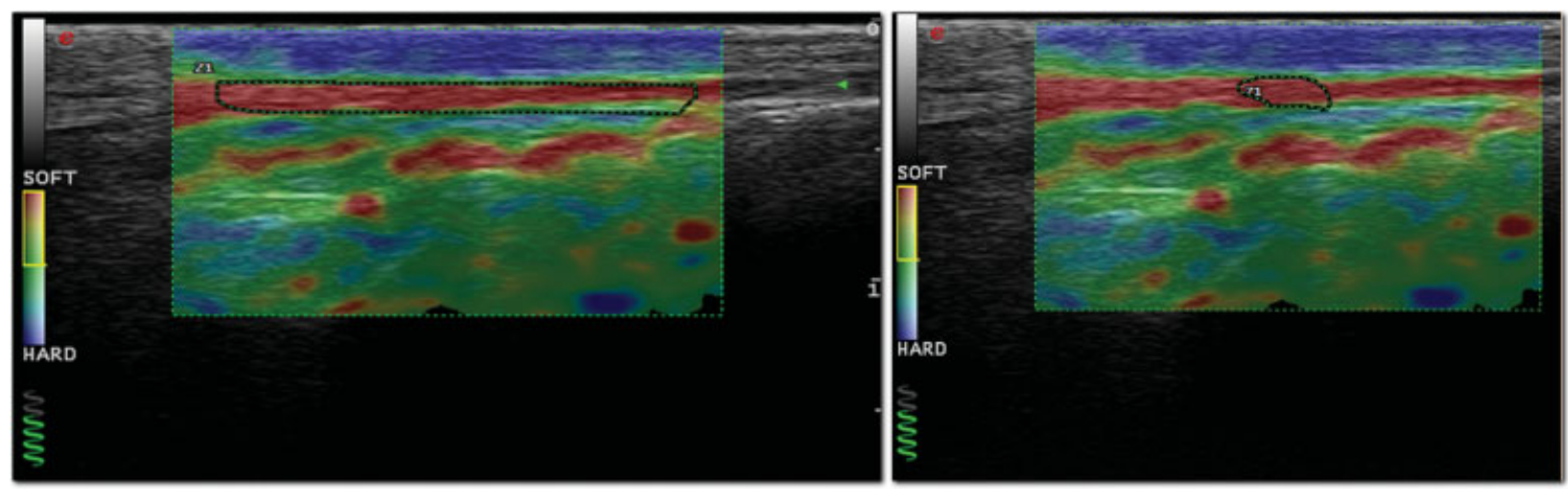

Fig. 3 Elastogram of a normal patellar ligament in a 2-year-old female Belgian shepherd dog. Measurements of the percentage of softness of the tissue (Elx-t\%sft) with two different types of regions of interest: entire main body of the ligament (left), central part of the ligament (right). 
Table 1 Categorical qualitative assessment of the patellar ligament elastogram with translation of the percentage of softness into a grading scale of 1-5

\begin{tabular}{|l|l|l|}
\hline Grading scale & \% of softness & Stiffness \\
\hline 1 & Elx-t\%sft $>70 \%$ & Soft \\
\hline 2 & $70 \%>$ Elx-t\%sft $>50 \%$ & Mostly soft \\
\hline 3 & $50 \%>$ Elx-t\%sft $>30 \%$ & Intermediate \\
\hline 4 & $30 \%>$ Elx-t\%sft $>20 \%$ & Mostly hard \\
\hline 5 & Elx-t\%sft $<20 \%$ & Hard \\
\hline
\end{tabular}

categorical qualitative assessment, and satisfactory elastographic images were obtained by each operator in all cases.

Qualitatively, patellar ligaments results were mainly red (soft), while the peritendinous tissue was mostly green (intermediate). The colour distribution was homogeneous over the entire ligament length. Mean ROI area was $15.2 \mathrm{~mm}^{2}$ for the entire main body of the ligament and $3 \mathrm{~mm}^{2}$ for the central part.

Overall, $89.3 \%$ of the patellar ligaments were graded as soft or mostly soft, while the remaining $10.7 \%$ were classified as intermediate. The mean softness value (Elx-t\%sft) \pm standard deviation was $94.9 \pm 9.3 \%$.

Repeatability was $86.2 \%$, with a weighted kappa of 0.64 (good), for the well-trained sonographer and $83.3 \%$, with a weighted kappa of 0.53 (moderate), for the senior student. Reproducibility was $86.2 \%$, with a weighed kappa of 0.65 (good). A good agreement (Cohen's Kappa $=0.65$ ) was found for both operators when comparing the two types of ROI measurement (entire main body of the ligament vs. central part).

\section{Discussion}

Based on our results, sonoelastography is a feasible imaging modality to evaluate tissue strain and softness/stiffness in the patellar ligament in this group of dogs. It is also a quick and safe procedure, taking approximately 5 minutes, without the use of sedation, contrast medium, or both.

Similar to the patellar ligament in humans, the normal canine patellar ligament showed a highly soft elastogram. ${ }^{11,41}$ Semi-quantitative analysis demonstrated that sonoelastography was moderately repeatable and reproducible, even with an inexperienced operator. The measurements in the small ROI drawn in the central part of the ligament were correlated to the measurements of the ROI including the entire main body of the ligament, depicting the ligament in this series of dogs as homogeneous.

Some difficulties in obtaining transversal sections, due to the lack of proper contact between the probe and the tissue examined, were observed. For this reason, only longitudinal sections were acquired. A further limitation was the size of the stifle. This technique was not feasible in small dogs, because to obtain diagnostic images, the footprint of the probe should be located between the apex of the patella and the tibial tuberosity. Moreover, during the study, dogs were evaluated only with the stifle in maximal passive flexion. In human medicine, it has been reported that the elasticity of the patellar ligament changes in function with the position of the knee, being highest in flexion and lowest in active forced extension, possibly reflecting the biomechanics of the knee joint. ${ }^{42}$ In fact, active forced extension is not easy to achieve in canine patients. Moreover, the correct positioning of the probe in extension may be difficult, due to the smaller dimensions of the canine stifle, as mentioned above.

Another limitation is represented by the initial cost of the dedicated software, which, however, is not very high when compared with other diagnostic techniques such as computed tomography or magnetic resonance imaging.

In this series of dogs, the patellar ligament was a highly elastic structure. This result is in contrast with studies on Achilles tendons of humans or digital flexor tendons in horses. $^{30-32,38,39}$ This discrepancy can be explained by the fact that the patellar ligament connects two fixed structures, namely the patella and the tibial tuberosity, while other tendons are attached to a hard structure at one side (bone) and to a soft and compliant structure, such as a muscle, to the other. The damper power of the muscle could explain the loss of the capacity of the system muscle-tendon to get back to the initial shape, while tendons connecting two bones appear highly elastic. ${ }^{41,42}$

Knowledge of the normal biomechanical properties of the patellar ligament with sonoelastography could be useful in assessing and monitoring dogs with patellar ligament desmopathy or injuries, such as after tibial plateau levelling osteotomy or tibial tuberosity advancement or trauma. Further studies are needed on a larger number of dogs with and without patellar ligament diseases.

In human literature, some inconsistencies on sonoelastographic features of patellar ligament tendinopathy exist. Athletes with unilateral patellar tendinopathy had stiffer ligament on the painful side than the non-painful side in a report, ${ }^{43}$ while in another article a soft patellar ligament has been associated with pain and functional deficit in volleyball players. ${ }^{33}$ This could be explained by the fact that the position of the knee during the examination changes the sonoelastographic features of the patellar ligament, as mentioned above. $^{42}$ In fact, the knee was flexed during elastography in the report that found the diseased patellar ligament stiffer. On the other hand, no information is given on knee position during sonoelastography in the other cited report, in which some inconsistencies in the interpretation of the colour strain map are also present. ${ }^{33}$ In fact, a hard ligament was described as 'elastic', while a soft ligament 'less elastic'. ${ }^{33}$ Moreover, some engagement of the quadriceps muscle by participants was possible in painful ligaments even in passive flexion. ${ }^{42}$

The precise assessment of the patellar ligament with quantitative measurement may improve the diagnostic accuracy and prognosis of tendinopathies and injuries, as it has already been shown with regard to the diseases of other tendons. ${ }^{32-34,39}$ Sonoelastography may be used during rehabilitation to modulate the exercise regimen in function of the elastic evolution of the healing ligament and may be used to monitor treatment effectiveness. 
In conclusion, sonoelastography of the canine patellar ligament is feasible, repeatable and reproducible in mediumand large-breed dogs. Normal canine patellar ligaments showed highly elastic biomechanical properties when evaluated with sonoelastography.

\section{Funding}

None.

\section{Conflict of Interest}

None.

\section{Author Contributions}

Angela P. Piccionello, Valeria Busoni, Alberto Salvaggio, Mattia Bonazzi, and Chiara Bergamino contributed to conception of study and study design. Angela P. Piccionello also contributed to data analysis and interpretation. Daniele Serrani and Antonella Volta contributed to conception of study, study design, acquisition of data, and data analysis and interpretation. All authors drafted and revised and approved the submitted manuscript.

\section{References}

1 Wolf RE, Scavelli TD, Hoelzler MG, Fulcher RP, Bastian RP. Surgical and postoperative complications associated with tibial tuberosity advancement for cranial cruciate ligament rupture in dogs: 458 cases (2007-2009). J Am Vet Med Assoc 2012;240(12):1481-1487

2 Kühn K, Ohlerth S, Makara M, Hässig M, Guerrero TG. Radiographic and ultrasonographic evaluation of the patellar ligament following tibial tuberosity advancement. Vet Radiol Ultrasound 2011;52(04):466-471

3 Stauffer KD, Tuttle TA, Elkins AD, Wehrenberg AP, Character BJ. Complications associated with 696 tibial plateau leveling osteotomies (2001-2003). J Am Anim Hosp Assoc 2006;42(01): 44-50

4 Pettitt R, Cripps P, Baker M, Hattersley R, Lorenz N, McConnell F. Radiographic and ultrasonographic changes of the patellar ligament following tibial tuberosity advancement in 25 dogs. Vet Comp Orthop Traumatol 2014;27(03):216-221

5 Boudrieau RJ. Tibial plateau leveling osteotomy or tibial tuberosity advancement? Vet Surg 2009;38(01):1-22

6 Hodgson RJ, O'connor PJ, Grainger AJ. Tendon and ligament imaging. Br J Radiol 2012;85:1157-1172

7 Pedersen M, Freedberg U, Langberg H. Sonoelastography as a diagnostic tool in the assessment of muscoloskeletal alteration: a systematic review. Ultraschall Med 2012;33:441-446

8 Ooi CC, Schneider ME, Malliaras P, Chadwick M, Connell DA. Diagnostic performance of axial-strain sonoelastography in confirming clinically diagnosed Achilles tendinopathy: comparison with B-mode ultrasound and color Doppler imaging. Ultrasound Med Biol 2015;41(01):15-25

9 Pedersen M, Fredberg U, Langberg H. Sonoelastography as a diagnostic tool in the assessment of musculoskeletal alterations: a systematic review. Ultraschall Med 2012;33(05):441-446

10 Pennick D, D'Anjou MA. Atlas of Small Animal Ultrasonography. 2nd ed. Hoboken, New jersey: John Wiley \& Sons; 2015:495

11 Carr JC, Hanly S, Griffin J, Gibney R. Sonography of the patellar tendon and adjacent structures in pediatric and adult patients. AJR Am J Roentgenol 2001;176(06):1535-1539

12 Muir P. Advances in the Canine Cruciate Ligament. 1st ed. John Wiley \& Sons and ACVS Foundation; 2010:117-121

13 Yamamoto Y, Yamaguchi S, Sasho T, et al. Quantitative ultrasound elastography with an acoustic coupler for Achilles tendon elasti- city: measurement repeatability and normative values. J Ultrasound Med 2016;35(01):159-166

14 Garra BS. Imaging and estimation of tissue elasticity by ultrasound. Ultrasound Q 2007;23(04):255-268

15 Klauser AS, Faschingbauer R, Jaschke WR. Is sonoelastography of value in assessing tendons? Semin Musculoskelet Radiol 2010;14 (03):323-333

16 Drakonaki EE, Allen GM, Wilson DJ. Ultrasound elastography for musculoskeletal applications. Br J Radiol 2012;85(1019): 1435-1445

17 Sadigh G, Carlos RC, Neal CH, Dwamena BA. Accuracy of quantitative ultrasound elastography for differentiation of malignant and benign breast abnormalities: a meta-analysis. Breast Cancer Res Treat 2012;134(03):923-931

18 Itoh A, Ueno E, Tohno E, et al. Breast disease: clinical application of US elastography for diagnosis. Radiology 2006;239(02): 341-350

19 Correas JM, Drakonakis E, Isidori AM, et al. Update on ultrasound elastography: miscellanea. Prostate, testicle, musculo-skeletal. Eur J Radiol 2013;82(11):1904-1912

20 Friedrich-Rust M, Ong MF, Herrmann E, et al. Real-time elastography for noninvasive assessment of liver fibrosis in chronic viral hepatitis. AJR Am J Roentgenol 2007;188(03):758-764

21 Shiraishi A, Hiraoka A, Aibiki T, et al. Real-time tissue elastography: non-invasive evaluation of liver fibrosis in chronic liver disease due to HCV. Hepatogastroenterology 2014;61(135): 2084-2090

22 Janssen J, Schlörer E, Greiner L. EUS elastography of the pancreas: feasibility and pattern description of the normal pancreas, chronic pancreatitis, and focal pancreatic lesions. Gastrointest Endosc 2007;65(07):971-978

23 Ragazzoni F, Deandrea M, Mormile A, et al. High diagnostic accuracy and interobserver reliability of real-time elastography in the evaluation of thyroid nodules. Ultrasound Med Biol 2012; 38(07):1154-1162

24 Dudea SM, Botar-Jid C, Dumitriu D, Vasilescu D, Manole S, Lenghel ML. Differentiating benign from malignant superficial lymph nodes with sonoelastography. Med Ultrason 2013;15 (02):132-139

25 Gong X, Wang Y, Xu P. Application of real-time ultrasound elastography for differential diagnosis of breast tumors. J Ultrasound Med 2013;32(12):2171-2176

26 Pallwein L, Mitterberger M, Struve P, et al. Real-time elastography for detecting prostate cancer: preliminary experience. BJU Int 2007;100(01):42-46

27 Xie M, Zhang X, Zhan J, Hua K. Application of real-time ultrasound elastography for discrimination of low- and high-grade serous ovarian carcinoma. J Ultrasound Med 2013;32(02):257-262

28 Thomas A, Kümmel S, Gemeinhardt O, Fischer T. Real-time sonoelastography of the cervix: tissue elasticity of the normal and abnormal cervix. Acad Radiol 2007;14(02):193-200

29 Drakonaki EE, Allen GM, Wilson DJ. Real-time ultrasound elastography of the normal Achilles tendon: reproducibility and pattern description. Clin Radiol 2009;64(12):1196-1202

30 De Zordo T, Fink C, Feuchtner GM, Smekal V, Reindl M, Klauser AS. Real-time sonoelastography findings in healthy Achilles tendons. Am J Roentgenol 2009;193(02):W134-W138

31 De Zordo T, Chhem R, Smekal V, et al. Real-time sonoelastography: findings in patients with symptomatic Achilles tendons and comparison to healthy volunteers. Ultraschall Med 2010;31 (04):394-400

32 Sconfienza LM, Silvestri E, Cimmino MA. Sonoelastography in the evaluation of painful Achilles tendon in amateur athletes. Clin Exp Rheumatol 2010;28(03):373-378

33 Ooi CC, Richards PJ, Maffulli N, et al. A soft patellar tendon on ultrasound elastography is associated with pain and functional deficit in volleyball players. J Sci Med Sport 2016;19(05): 373-378 


\section{Sonoelastographic Features of the Patellar Ligament Piccionello et al.}

34 Klauser AS, Miyamoto H, Tamegger M, et al. Achilles tendon assessed with sonoelastography: histologic agreement. Radiology 2013;267(03):837-842

35 LaCroix AS, Duenwald-Kuehl SE, Lakes RS, Vanderby RJr. Relationship between tendon stiffness and failure: a metaanalysis. J Appl Physiol (1985) 2013;115(01):43-51

36 Jeon S, Lee G, Lee SK, et al. Ultrasonographic elastography of the liver, spleen, kidneys and prostate in clinically normal beagle dogs. [corrected]Vet Radiol Ultrasound 2015;56: 425-431

37 White J, Gay J, Farnsworth R, Mickas M, Kim K, Mattoon J. Ultrasound elastography of the liver, spleen, and kidneys in clinically normal cats. Vet Radiol Ultrasound 2014;55(04): 428-434

38 Lustgarten M, Redding WR, Labens R, Morgan M, Davis W, Seiler GS. Elastographic characteristics of the metacarpal tendons in horses without clinical evidence of tendon injury. Vet Radiol Ultrasound 2014;55(01):92-101
39 Lustgarten M, Redding WR, Labens R, et al. Elastographic evaluation of naturally occurring tendon and ligament injuries of the equine distal limb. Vet Radiol Ultrasound 2015;56(06):670-679

40 Ozcan AN, Tan S, Tangal NG, et al. Real-time sonoelastography of the patellar and quadriceps tendons: pattern description in professional athletes and healthy volunteers. Med Ultrason 2016;18(03):299-304

41 Porta F, Damjanov N, Galluccio F, Iagnocco A, Matucci-Cerinic M. Ultrasound elastography is a reproducible and feasible tool for the evaluation of the patellar tendon in healthy subjects. Int J Rheum Dis 2014;17(07):762-766

42 Berko NS, Mehta AK, Levin TL, Schulz JF. Effect of knee position on the ultrasound elastography appearance of the patellar tendon. Clin Radiol 2015;70(10):1083-1086

43 Zhang ZJ, Ng GY, Lee WC, Fu SN. Changes in morphological and elastic properties of patellar tendon in athletes with unilateral patellar tendinopathy and their relationship with pain and functional disability. PLoS One 2014;9:1-9 